\title{
INTERVENÇÃO FARMACÊUTICA NA DETECÇÃO DE PROBLEMAS RELACIONADOS COM MEDICAMENTOS EM IDOSOS \\ INSTITUCIONALIZADOS: REVISÃO SISTEMÁTICA
}

PHARMACISTS' INTERVENTIONS IN MEDICATION-RELATED

PROBLEMS IN INSTITUTIONALIZED ELDERLY PEOPLE: A SYSTEMATIC REVIEW

\section{INTERVENCIÓN DE LA ATENCIÓN FARMACÉUTICA EN LA DETECCIÓN DE PROBLEMAS RELACIONADOS CON MEDICAMENTOS EN IDOSOS INSTITUCIONALIZADOS: REVISIÓN SISTEMÁTICA}

\author{
Ana Isabel Plácido (anaplacido@ipg.pt) * \\ Nídia Marante (niidii.a.mr@live.com.pt)** \\ Manuel Morgado (mmorgado@fcsaude.ubi.pt)*** \\ Maria Teresa Herdeiro (teresaherdeiro@ua.pt)**** \\ Fátima Roque (froque@ipg.pt)*****
}

\section{RESUMO}

\begin{abstract}
Este estudo tem como objetivo analisar Problemas Relacionados com Medicamentos (PRM) identificados por farmacêuticos em doentes idosos institucionalizados, através de uma revisão da literatura.

Para a elaboração deste estudo foi efetuada uma pesquisa na base de dados MEDLINE-PubMed. Foram selecionados todos os estudos publicados entre 1 de janeiro de 2000 e 31 de dezembro de 2017 que apresentassem resultados de deteção de PRM por farmacêuticos, em doentes idosos institucionalizados.

Após a aplicação dos critérios de inclusão e exclusão, foram incluídos, para análise, 12 estudos. A média de PRM identificados pelos farmacêuticos variou entre 1,92 e 15. Os principais PRM identificados nos diferentes estudos foram maioritariamente relacionados com problemas na seleção do medicamento e falta de monitorização ou monotorização inadequada.

Concluímos que o elevado número de PRM observado em idosos institucionalizados justifica a realização de intervenções que melhorem o uso dos medicamentos por parte desta população. Este estudo suporta também a tese de que a integração de farmacêuticos em equipas multidisciplinares de revisão de terapêutica pode contribuir para a minimização da ocorrência dos mesmos.
\end{abstract}

Palavras Chave: problemas relacionados com medicamentos, intervenção farmacêutica, revisão de terapêutica, farmacêutico, idoso institucionalizado. 


\section{ABSTRACT}

The aim of this review is to identify and summarize the main medication-related problems (DRPs) detected by pharmacists in institutionalized older adults.

The MEDLINE-PubMed library was searched for articles published between January 2000 and December 2017. Articles involving pharmacists in the identification of DRPs for institutionalized elderly people aged 65 and older were included.

Twelve studies were in accordance with the inclusion criteria. On average DRPs identified by pharmacists presented a mean of 1.92-15 DRPs.

The main cause of DRPs observed was related to medication selection followed by no or inappropriate outcome monitoring.

The high prevalence of DRPs in institutionalized older adults demonstrates the need for measures that improve medicine use in this population. These findings also support and encourage the integration of pharmacists into multidisciplinary teams underlining their role in clinical medication review.

Keywords: drug-related problems, pharmacists'interventions, medication review pharmacists, aged institutionalized.

\section{RESUMEN}

El artículo tiene como objetivo analizar problemas relacionados con los medicamentos (PRMs), identificados por farmacéuticos en ancianos institucionalizados a través de una revisión da literatura.

Para la preparación de este estudio se llevó a cabo una busca na base de datos MEDLINE-PubMed. Se seleccionaron todos los estudios disponibles entre el 1 de enero de 2000 y el 31 de diciembre de 2017 que presentaron resultados sobre problemas relacionados con los medicamentos (PRMs) en ancianos institucionalizados, identificados por farmacéuticos.

Doce estudios cumplieron con los criterios de inclusión. El valor medio de PRMs osciló entre 1,92 y 15. Los principales PRM estaban relacionados con la selección de medicamentos y con la falta de seguimiento o seguimiento inadecuado.

Los resultados obtenidos nos permiten concluir que el elevado número de PRM relacionados con la selección de medicamentos en personas mayores institucionalizadas son una realidad, y la intervención farmacéutica puede contribuir para la identificación y minimización da su aparición.

Palabras clave: problemas relacionados con medicamentos, intervención farmacéutica, revisión de terapias, farmacéuticos, personas mayores institucionalizadas.

* Unidade de Investigação para o Desenvolvimento do Interior, Instituto Politécnico da Guarda, Guarda, Portugal.

** Escola Superior de Saúde, Instituto Politécnico da Guarda, Guarda, Portugal.

*** Unidade de Investigação para o Desenvolvimento do Interior, Instituto Politécnico da Guarda, Guarda, Portugal. Escola Superior de Saúde, Instituto Politécnico da Guarda, Guarda, Portugal. Serviço Farmacêutico do Centro Hospitalar Universitário da Cova da Beira, Quinta do Alvito, 6200-251 Covilhã, Portugal. CICS-UBI - Centro de 
Investigação em Ciências Biomédicas Universidade da Beira Interior, Av. Infante D. Henrique, 6200-506 Covilhã, Portugal.

**** Departamento De Ciências Médicas e Instituto de Biomedicina iBiMED da Universidade de Aveiro campus universitário de santiago agra do crasto - edifício 30 3810-193 Aveiro.

***** Unidade de Investigação para o Desenvolvimento do Interior, Instituto Politécnico da Guarda, Guarda, Portugal. Escola Superior de Saúde, Instituto Politécnico da Guarda, Guarda, Portugal. CICS-UBI Centro de Investigação em Ciências Biomédicas Universidade da Beira Interior, Av. Infante D. Henrique, 6200-506 Covilhã, Portugal.

Submitted: 21 December 2018

Accepted: 8th July 2019 


\section{INTRODUÇÃO}

O uso de medicamentos na prevenção e tratamento de doenças é um inquestionável promotor da melhoria da qualidade de vida da população em geral, e da população idosa em particular. De facto, aproximadamente $33,3 \%$ de todas as prescrições médicas são de idosos (1). Estes números evidenciam não só o crescente envelhecimento da população mundial, cerca de $13 \%$ da população apresenta mais de 60 anos (2), mas também refletem o elevado número de medicamentos consumido por parte da população idosa. As múltiplas comorbidades associadas à idade tornam, por vezes, inevitável a prescrição de vários medicamentos, sendo que na população idosa institucionalizada o consumo de 5 ou mais medicamentos é frequente $(3,4)$. A polimedicação associada às alterações fisiológicas e ao declínio cognitivo característicos da idade poderá não só alterar a farmacocinética e farmacodinâmica dos fármacos mas também dificultar a otimização da terapêutica potenciando a ocorrência de problemas relacionados com os medicamentos (PRMs) (5).

De acordo com a Rede europeia de cuidados farmacêuticos (PCNE- Pharmaceutical Care Network Europe), PRMs são "Um Problema Relacionado a Medicamentos é um evento ou circunstância envolvendo tratamento com medicamentos que, interfere ou poderá interferir, nos resultados de saúde desejados." (6). Uma eficiente redução dos PRMs pode ser alcançada com a realização de intervenções que melhorem o uso de medicamentos por parte desta população (7). Vários estudos têm demonstrado não só o importante papel do farmacêutico na gestão da medicação mas também têm evidenciado que a inclusão destes profissionais em equipas de revisão terapêutica poderá potenciar o uso adequado de medicamentos e consequentemente a sua eficácia e segurança (8-11). Neste contexto, o objetivo deste trabalho foi apresentar uma revisão sistemática da literatura sobre estudos cujo objetivo é a identificação e classificação de PRMs por farmacêuticos na população idosa institucionalizada.

\section{MÉTODOS}

\section{Estratégias de Pesquisa e critérios de inclusão}

Foi efetuada uma pesquisa na base de dados MEDLINE-PubMed, utilizando a combinação dos seguintes termos de pesquisa "elderly" AND "drug related problems"; "older" AND "drug related problems", "elderly" AND "medication related problems" e "older" AND "medication related problems".

Foram incluídos todos os estudos publicados entre 1 de janeiro de 2000 e 31 de dezembro de 2017 que apresentavam os seguintes critérios de inclusão (1) escritos em Inglês, espanhol, português ou francês (2) cuja população alvo eram os doentes institucionalizados com idade igual ou superior a 65 anos (3) com revisão de medicação realizada por farmacêuticos ou equipas multidisciplinares que integrassem pelo menos um farmacêutico.

\section{Seleção dos estudos}

A seleção dos estudos incluídos foi realizada em duas etapas. Na primeira etapa dois investigadores (FR e AP) realizaram a leitura dos títulos e resumos de todos os estudos resultantes da pesquisa e excluíram aqueles que não obedeciam aos critérios de inclusão definidos. Na segunda etapa dois investigadores (NM e AP) realizaram a leitura completa de 
todos os artigos incluídos na etapa anterior e excluíram todos os que não se enquadravam nos critérios de inclusão. Sempre que se verificou discordância entre os dois investigadores, consultou-se um terceiro investigador (FR).

\section{Recolha de informação e análise de conteúdo}

Para cada um dos estudos um investigador recolheu informações relevantes. Subsequentemente um outro investigador verificou de forma independente os resultados recolhidos. A informação recolhida em cada um dos estudos encontrava-se relacionada com a localização do estudo, tipo de estudo, objetivo do estudo, caracterização da amostra, número de PRMs identificados, média de PRMs identificado por doente, sistema de classificação de PRMs utilizado. Nos estudos de intervenção o número de PRMs e a respetiva média foram utilizados os dados iniciais, antes da realização da intervenção. Nestes estudos para além destes dados também foi recolhida informação sobre os dados da intervenção. Os PRMs identificados em cada estudo foram reclassificados de acordo com o sistema de classificação Pharmaceutical Care Network Europe (PCNE) V8.01 (12).

\section{RESULTADOS}

Da pesquisa resultaram 948 estudos, e após aplicação dos critérios de inclusão e exclusão foram incluídos 12 para análise (Fig. 1).

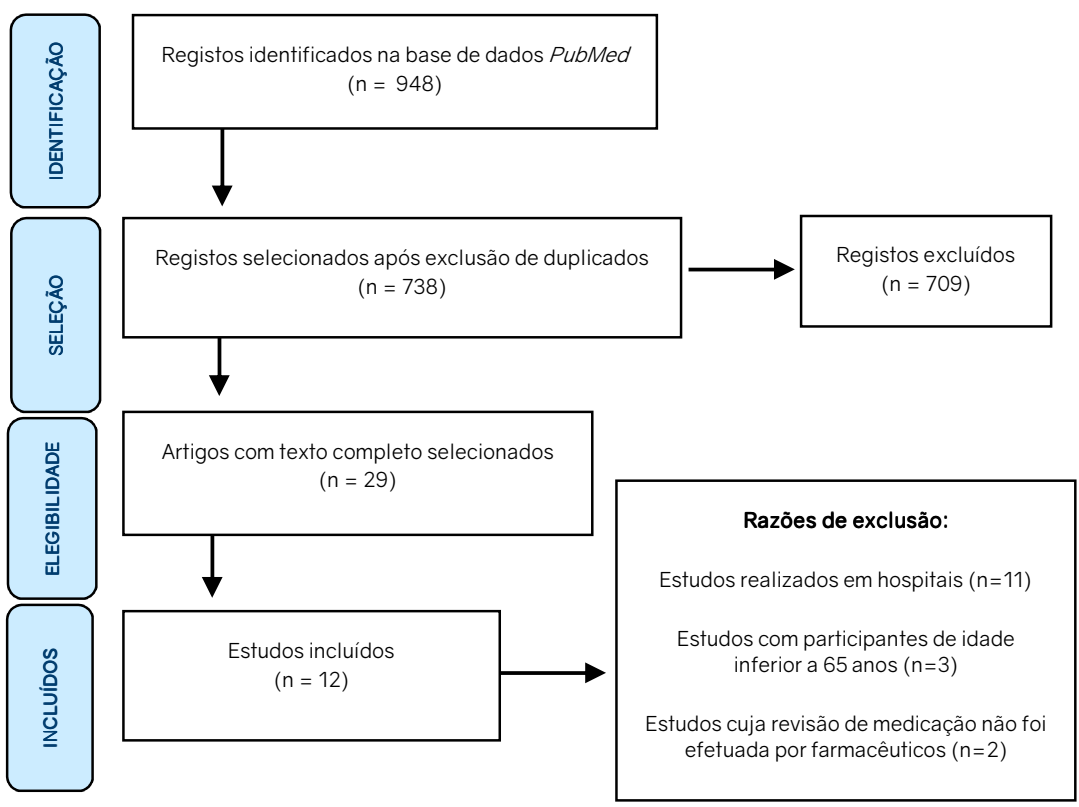

Figura 1 - Fluxograma da revisão da literatura

Na tabela 1 é possível observar que a maioria dos estudos incluídos foram desenvolvidos no continente Europeu $(3,13-20)(n=9)$, mais concretamente em Portugal $(n=2)(14,15)$, 
Espanha $(n=2)(13,19)$, Noruega $(n=2)(3,20)$, Holanda $(n=2)(17,18)$ e Suíça $(n=1)(16)$, três dos estudos foram realizados na Oceânia, mais propriamente na Austrália (21-23). Os estudos incluídos apresentavam uma população maioritariamente do sexo feminino, e com uma média de idades superior a 80 anos. O número de PRMs por utente apresentou, nos diferentes estudos variou entre 1,92 e 15. No continente Europeu, os dois estudos realizados em Espanha foram os que apresentaram um menor valor médio de PRMs por utente 1,92 (13) e 3,32(19). Os estudos realizados na Holanda apresentaram uma média de PRMs de 3,5 (18) e 3,8 (17), valor médio bastante próximo do de um dos estudo realizado na Suíça, cujo a media de PRMs foi de 3,7 (16). Em relação à media de PRMs obtida no estudo realizado na Noruega foi de 5,04 (20). Os estudos realizados na Austrália apresentaram valores médios de PRMs compreendidos entre 2,86 e 4,6 (21-23). 
Tabela 1- Características dos estudos incluídos

\begin{tabular}{|c|c|c|c|c|c|c|c|}
\hline $\begin{array}{l}\text { Autor } \\
\text { (ano) }\end{array}$ & $\begin{array}{l}\text { Continente } \\
\text { (pais) }\end{array}$ & $\begin{array}{l}\text { Tipo de } \\
\text { estudo }\end{array}$ & Objetivo do estudo & $\begin{array}{c}\text { Dimensão } \\
\text { da } \\
\text { amostra }\end{array}$ & $\begin{array}{l}\text { Caracterização da } \\
\text { amostra }\end{array}$ & $\begin{array}{c}\text { № de PRM } \\
\text { identificados (média } \\
\text { por utente) }\end{array}$ & $\begin{array}{l}\text { Sistema de } \\
\text { classificação de PRMs }\end{array}$ \\
\hline $\begin{array}{l}\text { Mestreset } \\
\text { al. (2017) } \\
\text { (13) }\end{array}$ & $\begin{array}{l}\text { Europa } \\
\text { (Espanha) }\end{array}$ & Observacional & $\begin{array}{c}\text { Descrever e avaliar o impacto da } \\
\text { intervenção farmacêutica na deteção de } \\
\text { PRMs }\end{array}$ & 8828 & $\begin{array}{l}\text { Média de idade: } 84,2 \\
\text { Feminino: } 70,2 \%\end{array}$ & $\begin{array}{l}4073 \\
(1,92)\end{array}$ & ASHP \\
\hline $\begin{array}{l}\text { da Costa et } \\
\text { al. } \\
(2016)(14)\end{array}$ & $\begin{array}{l}\text { Europa } \\
\text { (Portugal) }\end{array}$ & Observacional & $\begin{array}{l}\text { Determinar a prevalência de PRMs. } \\
\text { Avaliar a aceitação das intervenções } \\
\text { realizadas pelo farmacêutico }\end{array}$ & 126 & $\begin{array}{l}\text { Média de idade: } 84,8 \\
\text { Feminino: } 69 \%\end{array}$ & $\begin{array}{c}2109 \\
1002 \text { (grupo } \\
\text { intervencionado) } \\
\text { (mediana 14,5) }\end{array}$ & II Granada Consensus \\
\hline $\begin{array}{l}\text { Silva et al. } \\
(2015)(15)\end{array}$ & $\begin{array}{l}\text { Europa } \\
\text { (Portugal) }\end{array}$ & Observacional & $\begin{array}{c}\text { Avaliar a necessidade de implementação } \\
\text { de cuidados farmacêuticos em idosos } \\
\text { polimedicados }\end{array}$ & 31 & $\begin{array}{l}\text { Média de idade: } 81,65 \\
\text { Feminino: } 64,52 \%\end{array}$ & $\begin{array}{c}484 \\
\text { (mediana 15) }\end{array}$ & PCNE V6.2 \\
\hline $\begin{array}{c}\text { Gheewala et } \\
\text { al. } \\
(2014)(21)\end{array}$ & $\begin{array}{l}\text { Oceânia } \\
\text { (Austrália) }\end{array}$ & Observacional & $\begin{array}{c}\text { Avaliar o número e a natureza de PRMs } \\
\text { identificados } \\
\text { Avaliar as recomendações feitas pelos } \\
\text { farmacêuticos e a aceitação das mesmas } \\
\text { pelo medico }\end{array}$ & 847 & $\begin{array}{c}\text { Média de idade: } 84.9 \\
\text { Feminino: } 69 \%\end{array}$ & $\begin{array}{l}2712 \\
(3,2)\end{array}$ & DOCUMENT \\
\hline $\begin{array}{l}\text { Brulhart and } \\
\text { Wermeille } \\
(2011)(16)\end{array}$ & $\begin{array}{l}\text { Europa } \\
\text { (Suiça) }\end{array}$ & Observacional & $\begin{array}{l}\text { Avaliar um modelo de o sistema de } \\
\text { deteção de PRMs implementado em } \\
\text { idosos institucionalizados }\end{array}$ & 329 & $\begin{array}{l}\text { Média de idade: } 83.0 \\
\text { Feminino: } 76 \%\end{array}$ & $\begin{array}{l}1225 \\
(3,7)\end{array}$ & PCNE \\
\hline $\begin{array}{l}\text { Nishtala et } \\
\text { al. } \\
(2011)(22)\end{array}$ & $\begin{array}{l}\text { Oceânia } \\
\text { (Austrália) }\end{array}$ & Observacional & $\begin{array}{l}\text { Investigar o número e a natureza de PRMs } \\
\text { identificados por farmacêuticos. } \\
\text { Avaliar a aceitação e implementação pelo } \\
\text { medico das recomendações realizadas } \\
\text { por farmacêuticos }\end{array}$ & 500 & $\begin{array}{l}\text { Média de idade: } 84,0 \\
\text { Feminino: } 75 \%\end{array}$ & $\begin{array}{c}1433 \\
(2,86)\end{array}$ & n.d. \\
\hline $\begin{array}{c}\text { Halvorsen et } \\
\text { al. } \\
(2010)(3)\end{array}$ & $\begin{array}{l}\text { Europa } \\
\text { (Noruega) }\end{array}$ & Observacional & $\begin{array}{l}\text { Descrever uma equipa de intervenção } \\
\text { inovadora na deteção de PRMs }\end{array}$ & 142 & $\begin{array}{l}\text { Média de idade: } 86,9 \\
\text { Feminino: } 74,6 \%\end{array}$ & $\begin{array}{c}719 \\
(5,04)\end{array}$ & n.d. \\
\hline $\begin{array}{l}\text { Stafford et } \\
\text { al. } \\
(2009)^{2}(23)\end{array}$ & $\begin{array}{l}\text { Oceânia } \\
\text { (Austrália) }\end{array}$ & Observacional & $\begin{array}{l}\text { Analisar os PRMs identificados por } \\
\text { farmacêuticos em idosos } \\
\text { institucionalizados }\end{array}$ & 96 & $\begin{array}{l}\text { Média de idade: } 83.9 \\
\text { Feminino: } 67 \%\end{array}$ & $\begin{array}{c}376 \\
(4,6)\end{array}$ & DOCUMENT \\
\hline $\begin{array}{l}\text { Stuijtet al. } \\
(2008)(17)\end{array}$ & $\begin{array}{l}\text { Europa } \\
\text { (Holanda) }\end{array}$ & Observacional & $\begin{array}{l}\text { Avaliar o impacto de revisão de } \\
\text { medicação efetuada por farmacêuticos } \\
\text { nas prescrições realizadas a idosos }\end{array}$ & 30 & $\begin{array}{l}\text { Média de idade: } 85,8 \\
\text { Feminino: } 90 \%\end{array}$ & $\begin{array}{c}115 \\
(3,8)\end{array}$ & n.d. \\
\hline
\end{tabular}

${ }^{1}$ Resultados relativamente a idosos institucionalizados

ASHP- American Society of Hospital Pharmacists; PCNE- Pharmaceutical Care Network Europe; DOCUMENT- D.O.C.U.M.E.N.T classification system; n.d. não definido no estudo 


$$
\begin{gathered}
\text { EGITAN IA SCIENCIA } \\
N=25-2019 \quad \text { ISSN: } 1646-8848
\end{gathered}
$$

\begin{tabular}{|c|c|c|c|c|c|c|c|}
\hline $\begin{array}{l}\text { Finkers et al. } \\
\text { (2007)(18) }\end{array}$ & $\begin{array}{l}\text { Europa } \\
\text { (Holanda) }\end{array}$ & Observacional & $\begin{array}{l}\text { Investigar o número e a natureza de PRMs } \\
\text { identificados por farmacêuticos } \\
\text { hospitalares e médicos de instituições de } \\
\text { cuidados de idosos e avaliar as revisões } \\
\text { terapêuticas de medicação efetuadas por } \\
\text { farmacêuticos e médicos }\end{array}$ & 91 & $\begin{array}{l}\text { Média de idade: } 80,0 \\
\text { Feminino: } 68 \%\end{array}$ & $\begin{array}{l}323 \\
(3,5)\end{array}$ & BEDNURS study \\
\hline $\begin{array}{c}\text { Pastor } \\
\text { Climente et } \\
\text { al. } \\
(2007)(19)\end{array}$ & $\begin{array}{l}\text { Europa } \\
\text { (Espanha) }\end{array}$ & Intervenção & $\begin{array}{c}\text { Identificar os motivos de incumprimento } \\
\text { de terapêutica }\end{array}$ & 62 & $\begin{array}{l}\text { Média de idade: } 81.13 \\
\text { Feminino: } 79.03 \%\end{array}$ & $\begin{array}{c}206 \\
(3,32)\end{array}$ & n.d. \\
\hline $\begin{array}{c}\text { Ruths }(20) \\
\text { (2003) }\end{array}$ & $\begin{array}{l}\text { Europa } \\
\text { (Noruega) }\end{array}$ & Observacional & $\begin{array}{l}\text { Investigar o número e a natureza de PRMs } \\
\text { clinicamente relevantes. }\end{array}$ & 1324 & $\begin{array}{l}\text { Média de idade: } 85,2 \\
\text { Feminino: } 77 \%\end{array}$ & $\begin{array}{r}2445 \\
(1,85)\end{array}$ & n.d. \\
\hline
\end{tabular}


De acordo com o sistema de classificação da PCNE V8.01, o principal PRM identificado em 5 dos estudos $(15,19,20,22,23)$ foi um problema de segurança (P2), nomeadamente, a possível ocorrência de reações adversas (P2.1) (tabela 2). Dois estudos $(19,21)$ apresentaram como potencial PRM a eficácia do tratamento (P1) (mais concretamente um potencial problema com o efeito da farmacoterapia). Relativamente às causas associadas aos PRMs, oito dos estudos $(3,13,15,17,18,20,22,23)$ referiram que a seleção do medicamento foi a principal causa identificada no decorrer do estudo (C1), sendo que a não existência de indicação para a utilização do medicamento (C1.3), medicamentos inapropriados de acordo com os guidelines (C1.1) e a combinação inapropriada de medicamentos podendo ocorrer interações medicamentosas foram as subcategorias mais frequentemente observadas (C1.5). Dois estudos identificaram a falta de monitorização (C8.1) como a principal causa de PRMs $(21,23)$. Problemas de seleção da dose do medicamento (C2), por exemplo dosagem demasiado elevada (C2.2), foram também detetados com frequência nos diferentes estudos (16-18,2023) (tabela 2). Problemas referenciados com a forma farmacêutica inadequada (C2.1) foram identificados, num pequeno número de doentes, em 5 estudos $(13,15,16,21,23)$. PRMs relacionados com a duração do tratamento (C4) foram apontados apenas em dois dos estudos $(15,16)$. 


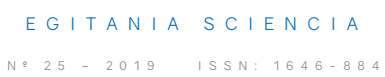

Tabela 2- Número de PRMs identificados nos estudos incluídos e respetivas causas segundo a PCNE

\begin{tabular}{|c|c|c|c|c|c|c|c|c|c|c|c|}
\hline \multicolumn{12}{|c|}{ "PHARMACEUTICAL CARE NETWORK EUROPE" (PCNE)- Classificação dos problemas relacionados com a medicação } \\
\hline \multirow[b]{2}{*}{$\begin{array}{l}\text { AUTOR } \\
\text { (ANO) }\end{array}$} & \multicolumn{3}{|c|}{ PROBLEMAS } & \multicolumn{8}{|c|}{ CAUSAS } \\
\hline & $\begin{array}{l}\text { Eficácia do } \\
\text { tratamento } \\
\text { (P1) }\end{array}$ & $\begin{array}{c}\text { Segurança } \\
\text { do } \\
\text { tratamento } \\
\text { (P2) }\end{array}$ & $\begin{array}{l}\text { Outras } \\
\text { (P3) }\end{array}$ & $\begin{array}{l}\text { Seleção do } \\
\text { medicamento } \\
\text { (C1) }\end{array}$ & $\begin{array}{c}\text { Forma } \\
\text { farmacêutica } \\
\text { (C2) }\end{array}$ & $\begin{array}{l}\text { Seleção } \\
\text { da dose } \\
\text { (C3) }\end{array}$ & $\begin{array}{l}\text { Duração do } \\
\text { tratamento } \\
\text { (C4) }\end{array}$ & $\begin{array}{l}\text { Dispensa } \\
\text { (C5) }\end{array}$ & $\begin{array}{l}\text { Uso do } \\
\text { medicamento } \\
\text { (C6) }\end{array}$ & $\begin{array}{c}\text { Relacionado } \\
\text { com o } \\
\text { doente } \\
\text { (C7) }\end{array}$ & $\begin{array}{l}\text { Outros } \\
\text { (C8) }\end{array}$ \\
\hline $\begin{array}{c}\text { Mestres et al. } \\
(2017)(13)\end{array}$ & 220 & 265 & 481 & 1980 & 679 & 344 & 0 & 0 & 0 & 94 & 0 \\
\hline $\begin{array}{c}\text { da Costa et al. } \\
(2016)(14)\end{array}$ & 0 & 0 & 0 & 1649 & 0 & 0 & 0 & 0 & 0 & 0 & 460 \\
\hline $\begin{array}{l}\text { Silva et al. } \\
(2015)^{1}(15)\end{array}$ & 72 & 240 & 93 & 284 & 3 & 35 & 16 & 0 & 28 & 0 & 37 \\
\hline $\begin{array}{c}\text { Gheewala et al. } \\
(2014)(21)\end{array}$ & 464 & 419 & 143 & 423 & 109 & 185 & 0 & 3 & 0 & 99 & 830 \\
\hline $\begin{array}{l}\text { Brulhart and } \\
\text { Wermeille } \\
(2011)(16)\end{array}$ & 0 & 2 & 374 & 272 & 45 & 233 & 199 & 0 & 0 & 79 & 0 \\
\hline $\begin{array}{l}\text { Nishtala et al. } \\
(2011)(22)\end{array}$ & 0 & 241 & 0 & 227 & 0 & 44 & 0 & 0 & 0 & 0 & 51 \\
\hline $\begin{array}{l}\text { Halvorsen et al. } \\
\text { (2010)(3) }\end{array}$ & 0 & 9 & 59 & 288 & 0 & 127 & 0 & 0 & 43 & 0 & 193 \\
\hline $\begin{array}{l}\text { Stafford et al. } \\
(2009)(23)\end{array}$ & 0 & 63 & 0 & 123 & 8 & 20 & 0 & 13 & 0 & 0 & 149 \\
\hline $\begin{array}{l}\text { Stuijt et al. } \\
(2008)(17)\end{array}$ & 0 & 18 & 19 & 50 & 0 & 28 & 0 & 0 & 0 & 0 & 0 \\
\hline $\begin{array}{c}\text { Finkers et al. } \\
(2007)(18)\end{array}$ & 0 & 10 & 66 & 236 & 0 & 11 & 0 & 0 & 0 & 0 & 0 \\
\hline $\begin{array}{c}\text { Pastor } \\
\text { Climente et al. } \\
(2007)^{2}(19)\end{array}$ & 17 & 7 & 0 & 37 & 0 & 0 & 0 & 34 & 15 & 96 & 0 \\
\hline $\begin{array}{c}\text { Ruths } \\
(2003)(20)\end{array}$ & 0 & 637 & 159 & 1085 & 0 & 361 & 0 & 0 & 0 & 0 & 203 \\
\hline
\end{tabular}

1 Os resultados originais encontravam-se em percentagem. Os PRMs foram calculados usando a seguinte forma \%PRMs x № total de PRMs / 100

${ }^{2}$ PRMs identificados antes da intervenção 
Em nove dos estudos incluídos, para além da identificação e caracterização dos principais PRMs identificados, foram também analisadas as recomendações feitas, pelo farmacêutico, aos médicos e qual a taxa de aceitação e de implementação das mesmas $(3,13-17,20,22,23)$ (tabela 3). Em 2 dos estudos $(13,14)$ foi possivel observar que mais de $50 \%$ das recomendações realizadas, por email carta ou telefone, não obtiveram qualquer tipo de resposta. A taxa de aceitação pelo médico, das intervenções sugerias pelo farmacêutico variou entre $8,7 \%(14)$ e $84 \%(13)$. A maioria dos estudos apresentam uma percentagem de implementação de intervenções propostas pelo farmacêutico superiores a $65 \%$, sendo que em dois estudos $(3,21)$ mais de $90 \%$ das intervenções propostas pelo farmacêutico foram aceites.

Tabela 3- características gerais dos estudos de análise de intervenções realizadas por farmacêuticos

\begin{tabular}{|c|c|c|c|c|c|}
\hline $\begin{array}{l}\text { Autor } \\
\text { (ano) }\end{array}$ & $\begin{array}{c}\text { Utentes } \\
\text { intervencionados }\end{array}$ & $\begin{array}{l}\text { Intervenções } \\
\text { propostas ao } \\
\text { médico }\end{array}$ & $\begin{array}{l}\text { \% de } \\
\text { respostas }\end{array}$ & $\begin{array}{l}\text { \% Intervenções } \\
\text { implementadas }\end{array}$ & $\begin{array}{l}\text { \% intervenções } \\
\text { implementada } \\
\text { relativamente às } \\
\text { recomendações } \\
\text { com resposta }\end{array}$ \\
\hline $\begin{array}{c}\text { Mestres et } \\
\text { al. } \\
(2017)^{1}(13)\end{array}$ & 2119 & 4073 & $44 \%$ & $\begin{array}{c}37 \% \\
\text { (entre } 57.9 \text { e } \\
28.5 \% \text { nos } 10 \\
\text { PRMs mais } \\
\text { frequentes, a \% é } \\
\text { dependente do } \\
\text { tipo de PRM ) }\end{array}$ & $84 \%$ \\
\hline $\begin{array}{c}\text { da Costa et } \\
\text { al. } \\
(2016)(14)\end{array}$ & 63 & 539 & 45,2 & $2,8 \%$ & 8,7 \\
\hline $\begin{array}{c}\text { Gheewala et } \\
\text { al. } \\
(2014)(21)\end{array}$ & 830 & 3054 & - & 83,8 & - \\
\hline $\begin{array}{l}\text { Brulhart and } \\
\text { Wermeille } \\
\text { (2011)(16) }\end{array}$ & 847 & 1228 & - & $93 \%$ & - \\
\hline $\begin{array}{c}\text { Nishtala et } \\
\text { al. } \\
(2011)(22)\end{array}$ & 480 & 1433 & - & 72,5 & - \\
\hline $\begin{array}{c}\text { Halvorsen et } \\
\text { al. } \\
(2010)(3)\end{array}$ & 140 & 504 & - & 93,7 & - \\
\hline $\begin{array}{l}\text { Stuijtetal. } \\
(2008)(17)\end{array}$ & 30 & 115 & - & 67,8 & - \\
\hline $\begin{array}{l}\text { Finkers et } \\
\text { al. } \\
(2007)(18)\end{array}$ & 80 & 323 & - & 49,2 & - \\
\hline $\begin{array}{c}\text { Pastor } \\
\text { Climente et } \\
\text { al. } \\
(2007)(19)\end{array}$ & 62 & 39 & - & $68 \%$ & - \\
\hline
\end{tabular}

\section{DISCUSSÃO}

Na ultima década vários estudos têm sustentado a tese de que a integração de farmacêuticos nas equipas de cuidados de saúde primário poderá potenciar: a melhoria dos resultados clínicos do doente; a prevenção de PRMs; a redução de custos de saúde; d- uma melhor gestão de recursos humanos (24-31). A pertinência das observações dos referidos estudos estimularam, em alguns países, o desenvolvimento de medidas promotoras da integração de

\footnotetext{
${ }^{1}$ Foram considerados exclusivamente os resultados relativamente aos idosos institucionalizados.
} 
farmacêuticos em equipas multidisciplinares de acompanhamento de doentes no sistema de saúde $(27,32,33)$. No entanto, a maioria destes estudos foi realizado em ambiente de cuidado de saúde primário, internamento ou ambulatório, sendo escassos os estudos realizados em idosos institucionalizados. Para além disso e de acordo com o nosso conhecimento não existe até ao momento nenhum estudo que reveja sistematicamente as intervenções farmacêuticas na deteção de PRMs em idosos institucionalizados. Neste contexto este trabalho assume um papel essencial na promoção do conhecimento relacionado com PRMs em idosos institucionalizados para o posterior desenvolvimento de estratégias que promovam a redução de PRMs nesta população. Apesar da heterogeneidade dos estudos incluídos neste trabalho, os diferentes estudos demonstram que os idosos institucionalizados para além de se encontrarem polimedicados apresentam um valor médio de PRMs por estudo bastante elevado, que varia entre aproximadamente 2 PRMs e 15 PRMs por utente. Esta observação é corroborada por vários estudos que demonstram que a polimedicação, situação recorrente em idosos institucionalizados, favorece a ocorrência de PRMs (34-37). O elevado número de PRMs é preocupante, e demonstra a necessidade de implementação de medidas eficazes de deteção e correção dos mesmos. Neste contexto a integração de farmacêuticos em equipas multidisciplinares de revisão de terapêutica poderá ser uma mais valia. De facto vários estudos demonstram que, em contexto hospitalar, os farmacêuticos são profissionais qualificados e capacitados para a realização/ implementação de estratégias que visam melhorar o estado de saúde do doente e potenciar a gestão humanística e económica associada aos cuidados de saúde (24-31). Atualmente, existem vários sistemas de classificação de PRMs, neste estudo e de forma a podermos estabelecer uma comparação de PRMS optámos por classificar todos os PRMs de acordo com o sistema de classificação proposto pela rede europeia de seguimento farmacoterapêutico PCNE 8.01. De acordo com o referido sistema de classificação, o PRM mais frequentemente observado nos diferentes estudos foi a seleção do medicamento. Esta observação encontra-se em concordância com a literatura (34-37). Vários estudos demonstram que a polimedicação torna os idosos mais suscetíveis a PRMs relacionados com a seleção do fármaco (34-37), nomeadamente ocorrência de interações medicamentosas e possíveis ocorrências de reações adversas (36). A frequente ocorrência deste tipo de PRMs poderá dever-se a vários fatores nomeadamente a alterações farmacocinéticas associadas à idade ou subjacentes à própria doença ou ainda ao facto de doentes se encontrarem polimedicados o que potencia a ocorrência de interações entre fármacos. Todos estes factores dificultam a optimização das terapêuticas e potenciam a ocorrência de potenciais PRMs (5). Neste contexto e de forma a diminuir este tipo de PRMs a existência de equipas multifacetadas de profissionais de saúde atentos e com formação em PRMs é essencial não só para a realização da correta monitorização, mas também para gestão e revalidação dos riscos/ benefícios da farmacoterapia (8). Os resultados referentes à aceitação de intervenções sugeridas pelo farmacêutico, devem ser analisados com precaução, visto que os referidos resultados são dependentes de vários fatores extrínsecos ao estudo, nomeadamente: as políticas internas do país onde o estudo foi realizado, o ano em que o estudo foi realizado, o desenho do processo de revisão de medicação, a comunicação entre os profissionais de saúde, entre outros fatores. Na realização deste estudo observou-se que a maioria dos estudos apresentava uma percentagem de implementação de propostas sugeridas pelo farmacêutico superior a 50\% $(3,16,17,19,21,22)$. A baixa taxa de aceitação de intervenções propostas pelo farmacêutico observada no estudo realizado em Portugal poder-se-á encontrar relacionados com vários fatores, nomeadamente a falta de comunicação entre os diferentes profissionais de saúde, ou tendo em consideração o facto de existir uma correlação inversa entre o número de intervenções propostas e o número de aceitações, com o elevado número de recomendações propostas a cada um dos médicos (14). A elevada aceitação de intervenções observada nos estudos realizados na Austrália, Noruega e Suíça $(27,33,38)$ 
poderá dever-se ao facto de nos últimos anos e com o objetivo de melhorar os indicadores clínicos económicos e humanísticos, estes países terem promovido politicas de integração de farmacêuticos em equipas multidisciplinares de profissionais de saúde $(27,33)$. Na realização deste estudo não foi possível quantificar o benefício da integração de farmacêuticos em equipas de revisão de terapêutica, no entanto os estudos incluídos neste trabalho destacam o seu papel na identificação e redução de PRMs.

\section{CONCLUSÃo}

Os PRMs na população idosa institucionalizada são uma realidade pelo que é importante que haja uma crescente consciencialização da sociedade através da implementação de intervenções educativas e sociais que capacitem profissionais de saúde, utentes e seus cuidadores para um melhor uso de medicamentos e que facilitem a comunicação entre os profissionais de saúde e também com os utentes. O empoderamento e capacitação de doentes idosos e seus cuidadores é fundamental para uma redução dos PRMs não só em idosos institucionalizados, mas na população idosa em geral. O elevado número de PRMs relacionados com a seleção do medicamento, sugere a necessidade de realização de estudos cujo foco seja a analise de sistemas implementação de sistemas de suporte a apoio à decisão clinica em idosos institucionalizados.

\section{Agradecimentos}

Este trabalho foi financiado no âmbito dos projetos MedElderly [SAICT-POL/23585/2016], co-financiado pela fundação para a Ciência e Tecnologia através de fundos FEDER e Centro 2020, Centro-01-0145-FEDER-023585 e APIMedOIder [PTDC/MED-FAR/31598/2017], cofinanciado pelo Programa Operacional Competitividade e Internacionalização ( $\mathrm{POCl}$ ) na sua componente FEDER/FNR, POCI-01-0145-FEDER-031598 e Fundação para a Ciência e Tecnologia, na sua componente de orçamento de estado.

\section{BIBLIOGRAFIA}

1. Carmona-Torres JM, Cobo-Cuenca AI, Recio-Andrade B, Laredo-Aguilera JA, Martins MM, Rodríguez-Borrego MA. Prevalence and factors associated with polypharmacy in the older people: 2006-2014. J Clin Nurs. 2018;

2. United Nations, Department of Economic and Social Affairs PD (2017). World population prospects: the 2012 Revision, Volume II: demographic profiles. World Population Prospects: The 2017 Revision, Volume II: Demographic Profiles (ST/ESA/SER.A/400). 2017.

3. Halvorsen KH, Ruths S, Granas AG, Viktil KK. Multidisciplinary intervention to identify and resolve drug-related problems in Norwegian nursing homes. Scand J Prim Health Care. 2010;

4. Golchin N, Isham L, Meropol S, Vince A, Frank S. Polypharmacy in the elderly. J Res Pharm Pract. 2015;

5. Kim J, Parish AL. Polypharmacy and Medication Management in Older Adults. Vol. 52, Nursing Clinics of North America. 2017. p. 457-68.

6. Pharmaceutical Care Network Europe Foundation. No Title [Internet]. [cited 2019 Mar 14]. Available from: https://www.pcne.org/working-groups/2/drug-related-problem-classification

7. Hayashi Y, Godai A, Yamada M, Yoshikura N, Harada N, Koumura A, et al. Reduction in the 
numbers of drugs administered to elderly in-patients with polypharmacy by a multidisciplinary review of medication using electronic medical records. Geriatr Gerontol Int. 2017;

8. Kallio S, Kumpusalo-Vauhkonen A, Järvensivu T, Mäntylä A, Pohjanoksa-Mäntylä M, Airaksinen $M$. Towards interprofessional networking in medication management of the aged: current challenges and potential solutions in Finland. Scand J Prim Health Care. 2016;

9. Hanlon JT, Lindblad Cl, Gray SL. Can clinical pharmacy services have a positive impact on drugrelated problems and health outcomes in community-based older adults? American Journal Geriatric Pharmacotherapy. 2004.

10. Alldred DP, Raynor DK, Hughes C, Barber N, Chen TF, Spoor P. Interventions to optimise prescribing for older people in care homes. Cochrane database Syst Rev. 2013;

11. AG Z, DP A, DR P, DK R, Freemantle N, Eastaugh J, et al. Clinical medication review by a pharmacist of elderly people living in care homes--randomised controlled trial. Age Ageing. 2006;

12. Pharmaceutical Care Network Europe Foundation. PCNE Classification of drug-related problems. Tidsskr Nor Laegeforen. 2007;127(23):3073-6.

13. Mestres C, Agustí A, Hernandez M, Puerta L, Llagostera B. Pharmacist Intervention Program at Different Rent Levels of Geriatric Healthcare. Pharmacy. 2017;

14. da Costa FA, Silvestre L, Periquito C, Carneiro C, Oliveira P, Fernandes Al, et al. Drug-Related Problems Identified in a Sample of Portuguese Institutionalised Elderly Patients and Pharmacists' Interventions to Improve Safety and Effectiveness of Medicines. Drugs - Real World Outcomes. 2016;

15. Silva C, Ramalho C, Luz I, Monteiro J, Fresco P. Drug-related problems in institutionalized, polymedicated elderly patients: opportunities for pharmacist intervention. Int J Clin Pharm. 2015;

16. Brulhart MI, Wermeille JP. Multidisciplinary medication review: Evaluation of a pharmaceutical care model for nursing homes. Int J Clin Pharm. 2011;

17. Stuijt CCM, Franssen EJF, Egberts ACG, Hudson SA. Appropriateness of prescribing among elderly patients in a Dutch residential home: Observational study of outcomes after a pharmacistled medication review. Drugs and Aging. 2008;

18. Finkers F, Maring JG, Boersma F, Taxis K. A study of medication reviews to identify drug-related problems of polypharmacy patients in the Dutch nursing home setting. J Clin Pharm Ther. 2007;

19. Pastor Climente IP, Ortiz De Urbina Sandomingo V, Pérez Escoto I, Quintana Vargas I, Moreno Miralles A, Martínez Martínez M. The introduction of a programme to improve the treatment compliance of institutionalised elderly patients. Farm Hosp. 2007;

20. Ruths S. Multidisciplinary medication review in nursing home residents: what are the most significant drug-related problems? The Bergen District Nursing Home (BEDNURS) study. Qual Saf Heal Care. 2003;

21. Gheewala PA, Peterson GM, Curtain CM, Nishtala PS, Hannan PJ, Castelino RL. Impact of the Pharmacist Medication Review Services on Drug-Related Problems and Potentially Inappropriate Prescribing of Renally Cleared Medications in Residents of Aged Care Facilities. Drugs and Aging. 2014;

22. Nishtala PS, McLachlan AJ, Bell JS, Chen TF. A retrospective study of drug-related problems in Australian aged care homes: Medication reviews involving pharmacists and general practitioners. J Eval Clin Pract. 2011;

23. Stafford AC, Tenni PC, Peterson GM, Jackson SL, Hejlesen A, Villesen C, et al. Drug-related problems identified in medication reviews by Australian pharmacists. Pharm World Sci. 2009;

24. Gagnon A, Jin M, Malak M, Bednarowski K, Feng L, Francis-Pringle S, Lu S, Mallin A, SkokovicSunjic D VA. Pharmacists Managing People with Diabetes in Primary Care: 10 Years of Experience at the Hamilton Family Health Team. Can J Diabetes. 2017;41(6):576-9. 
25. Hazen ACM, de Bont AA, Leendertse AJ, Zwart DLM, de Wit NJ, de Gier JJ BM. How Clinical Integration of Pharmacists in General Practice has Impact on Medication Therapy Management: A Theory-oriented Evaluation. Int J Integr Care. 2019;19(1)(1).

26. Hazen ACM, de Bont AA, Boelman L, Zwart DLM, de Gier JJ, de Wit NJ, et al. The degree of integration of non-dispensing pharmacists in primary care practice and the impact on health outcomes: A systematic review. Research in Social and Administrative Pharmacy. 2018.

27. Lourenço AFOCD. Innovation in Health: first contribution to the development of a New Practice Model for Clinical Pharmacists in Primary Healthcare [Internet]. Coimbra; 2018. Available from: http://hdl.handle.net/10316/84625

28. Tan ECK, StewartK, Elliott RA, George J. Integration of pharmacists into general practice clinics in Australia: The views of general practitioners and pharmacists. Int J Pharm Pract. 2014;

29. Tan ECK, StewartK, ElliottRA, George J. Pharmacist services provided in general practice clinics: A systematic review and meta-analysis. Research in Social and Administrative Pharmacy. 2014.

30. Dolovich L, Pottie K, Kaczorowski J, Farrell B, Austin Z, Rodriguez C, et al. Integrating family medicine and pharmacy to advance primary care therapeutics. Clin Pharmacol Ther. 2008;

31. Freeman C, Cottrell WN, Kyle G, Williams I, Nissen L. Integrating a pharmacist into the general practice environment: Opinions of pharmacists, general practitioners, health care consumers, and practice managers. BMC Health Serv Res. 2012;

32. NHS England. Clinical Pharmacists in General Practice Pilot [Internet]. 2017. Available from: https://www.england.nhs.uk/commissioning/primary-care-comm/gp-action-plan/cp-gppilot/

33. Kroneman M, Boerma W, van den Berg M, Groenewegen P, de Jong J, van Ginneken E. Netherlands: Health System Review. Health Syst Transit. 2016;

34. Bao Y, Shao H, Bishop TF, Schackman BR, Bruce ML. Inappropriate medication in a national sample of US elderly patients receiving home health care. J Gen Intern Med. 2012;

35. Cannon KT, Choi MM, Zuniga MA. Potentially inappropriate medication use in elderly patients receiving home health care: A retrospective data analysis. Am J Geriatr Pharmacother. 2006;

36. Maher RL, Hanlon J, Hajjar ER. Clinical consequences of polypharmacy in elderly. Expert Opin Drug Saf. 2014;

37. Prithviraj GK, Koroukian S, Margevicius S, Berger NA, Bagai R, Owusu C. Patient characteristics associated with polypharmacy and inappropriate prescribing of medications among older adults with cancer. J Geriatr Oncol. 2012;

38. H Benson, C Lucas, SI Benrimoj, W Kmet. Williams KA. Pharmacists in general practice: recommendations resulting from team-based collaborative care. Aust J Prim Health. 2018;24(6):448-54.

39. Hazen ACM, de Bont AA, Leendertse AJ, Zwart DLM, de Wit NJ, de Gier JJ BM. How Clinical Integration of Pharmacists in General Practice has Impact on Medication Therapy Management: A Theory-oriented Evaluation. Int J Integr Care. 2019;19(1)(1). 
